\title{
Pyroelectric ceramics and thin films for applications in uncooled infra-red sensor arrays
}

\author{
Roger W. Whatmore ${ }^{1}$, Qi Zhang ${ }^{2}$, Christopher P. Shaw ${ }^{2}$, Robert A. Dorey ${ }^{2}$ and Jeffery \\ R. Alcock ${ }^{2}$ \\ 1. Tyndall National Institute, Lee Maltings, Cork, Ireland \\ 2. Cranfield University, Cranfield, Bedfordshire, MK43 0AL, UK
}

\begin{abstract}
Pyroelectric infra-red detector arrays provide an attractive solution to the problem of collecting spatial information in the far IR. They are only sensitive to changes in the IR flux and are well suited to sensing movements of people. The applications of low cost arrays with limited (few hundred) elements for people sensing \& radiometry will be illustrated. The performances of uncooled pyroelectric arrays are ultimately driven by the materials used. For this reason, continuous improvements in materials technology and figures-of-merit are important. The performance of dense, bulk pyroelectric ceramics has not increased for several years, but nevertheless it is possible to obtain significant improvements in performance thorugh the use of tape-cast, functionallygradient materials (FGMs) in which controlled porosity is used to control the permittivity and heat capacity of the material. A model for the performance of such a material will be presented, and compared with experimentally-determined properties of FGMs based on a modified-PZT pyroelectric ceramic. The use of ferroelectric thin films is offering considerable potential for low cost \& high performance. It will be shown that the introduction of controlled amounts of porosity can have a significant positive effect on the relevant pyroelectric figure-of-merit.
\end{abstract}

Keywords: Pyroelectrics, Infra-red, arrays, ferroelectric, ceramics, thin films, functionally gradient materials 


\section{Introduction}

Pyroelectric IR detectors have been used for many years in applications ranging from intruder sensing through environmental monitoring to flame and fire detection [1]. In the last 15 years there has been a growth of interest in using 2D arrays for uncooled thermal imaging [2,3]. In such arrays, the resolution of the image obtained is determined by the number of elements in the detector, and for these large numbers of very small pyroelectric elements are needed. Such devices have come under severe competition in the last few years from uncooled resistance bolometer arrays [4], to the extent that there are few commercial systems which now use pyroelectrics for themal imaging. However, the characteristics of pyroelectric detectors, whereby they will give an output only for a change in infra-red flux, still makes them ideally-suited to the intruder and flame detection application. In this scenario, no complex signal processing is needed to detect the presence of a warm target against a visually-cluttered, but static, background. Pyroelectric detectors are still pre-eminent in these applications, particularly as single, dual and quad-element detectors. Recently, it has been demonstrated that low element count arrays (e.g. 16x16) can be very usefully applied to many applications [5], such as people-counting and queue-length monitoring. In these applications, high image resolution is not required and may, indeed, be seen as an invasion of privacy by the subject. In one application (SIMBAD [6]) the device has been applied to a fall detector, intended to enhance the quality of life of older persons while affording a greater sense of security and facilitating independent living. This paper reviews the requirements of bulk ceramic and thin film materials for pyroelectric detectors and some recent developments in the area.

\section{Pyroelectric Devices and Ceramic Materials}


Fig. 1 shows a schematic diagram of a pyroelectric infra-red detector. A thin piece of pyroelectric (the “element”) is electroded on its major faces and exposed to infra-red radiation, which produces a temperature change in the element. The resulting pyroelectric current, $i_{p}$ which flows through the gate-bias resistor $R_{G}$ will be given by $i_{p}=A p d T / d t . \quad p$ is the pyroelectric coefficient and $d T / d t$ is the rate of change of the element's temperature with time. Thus, pyroelectric devices are coupled to the changes of any input energy flux - such as infra-red radiation - which generate a change in element temperature with time.

The physics of pyroelectric detectors has been reviewed previously [1]. If $C_{E}>>C_{A}$ the voltage response is proportional to a figure-of-merit (FoM) $F_{V}=\frac{p}{c^{\prime} \varepsilon \varepsilon_{o}}$ where $\mathrm{c}^{\prime}=$ volume specific heat, $\varepsilon_{o}$ is the permittivity of free space and $\varepsilon$ the relative permittivity of the pyroelectric material. If $C_{A}>>C_{E}$ the voltage response is proportional to $F_{i}=\frac{p}{c^{\prime}}$, which would apply to maximising the response of very small elements. Hence, it can be seen that the choice of the appropriate material will depend both on the amplifier to be used and on the size of the detector element. Small area detector elements will tend to favour high dielectric constant materials and vice versa, to produce a good match between $C_{A}$ and $C_{E}$. It is evidently insufficient to consider only the response of a detector when analysing its usefulness for a particular application. It is generally necessary to analyse both intrinsic and extrinsic noise signals and compare them with the response. A figure-of-merit frequently used in the discussion of detector performance is the specific detectivity $D^{*}$. To maximise $D^{*}$ in the region where 
Johnson Noise due to the loss tangent $(\tan \delta)$ of the pyroelectric material dominates, it is desirable to maximise $F_{D}=\frac{p}{c^{\prime} \sqrt{\varepsilon \varepsilon_{o} \tan \delta}}$.

The structure of a typical pyroelectric array is shown schematically in Fig. 2. These arrays are microsystem-type devices in which each pyroelectric element is interfaced to its own integrated FET amplifier, these being linked by arrays of switches for multiplexing. The devices have been made possible through the flip-chip hybridisation of thin pyroelectric ceramic sensor layers with silicon application specific integrated circuits (ASICs) - known as read-out integrated circuits (ROICs) [2]. In use, the devices are placed at the focus of an infra-red lens, capable of focusing radiation in the 8 to 12 micron wavelength range, usually made of germanium. Because pyroelectric devices are only sensitive to changes in temperature, and hence IR intensity, the array can be used in two ways. If the array is simply placed in the focal plane of the array, it will not be sensitive to static scenes or objects which do not move. This means that the system can be used in applications where there is a need to sense moving warm objects against a relatively unchanging background. These include people counting and sensing systems for use in retail applications. Stogdale et al [7] have reported the use of a 16x16 array [8] in a system for people counting and queue length monitoring applications. Fig. 3 illustrates a visible (right) and infra-red (left) view of people walking beneath a sensor system using this array. The array is low resolution and is not used to create an image, but is linked to an automatic system which can count people. Recently, such a system used for retail queue-length monitoring has won an award [9]. If a means for modulating the radiation, such as a rotating chopper [10], is placed in front of such an array, it can be used for producing thermal images. 


\section{Pyroelectric Ceramics}

There are two main classes of pyroelectric ceramics that are used commercially in infrared detector arrays: ceramics with a Curie temperature $\mathrm{T}_{\mathrm{C}}$ well above ambient (typically $>200^{\circ} \mathrm{C}$ ), so that the pyroelectric coefficient is reversible and stable and ceramics with a $\mathrm{T}_{\mathrm{C}}$ around room temperature, for which the pyroelectric coefficient must be stabilised by the application of a DC electric field. The latter group of devices are frequently referred to as dielectric bolometers [10].

The most widely used high $\mathrm{T}_{\mathrm{C}}$ pyroelectric ceramics are based on modified lead zirconate (PZ) [11,12 \&13] or modified lead titanate [14]. The morphotropic phase boundary compositions of the PZT system are not used for pyroelectric applications because they have high permittivities. The FoM for a selection of typical pyroelectric ceramics are shown in Table 1.

In many cases, it is desirable to control the electrical conductivity of the pyroelectric ceramic. This will determine the electrical time constant $\left(\tau_{\mathrm{E}}\right)$ of the detector element ( $\tau_{E}=R_{G}\left(C_{E}+C_{A}\right)$ ) by fixing $R_{G}$ in Fig. 1. (Fixing $R_{G}$ also fixes the bias point of the FET.) $\tau_{\mathrm{E}}$ will also determine the time that the array takes to "settle" when the system temperature is changed. It has been shown $[15,16]$ that uranium is a highly effective dopant for conductivity control in two families of modified lead zirconate ceramics and that the behaviour can be modelled in terms of hopping conduction of carriers, probably electrons, between localized sites in the ceramics. This model predicts that $\log \sigma$ (where $\sigma$ is the ceramic conductivity) is proportional to $\mathrm{z}^{-1 / 3}$, where $\mathrm{z}$ is the amount of U-dopant added. Fig. 4 [16] illustrates this for these two ceramic families. A similar type of 
behaviour is followed for Cr-doping [17], except that this would be expected to act as an acceptor.

It can be seen that the basic figures-of-merit of the ceramic materials listed in Table 1 are quite similar, and indeed it is hard to improve on these by simply changing ceramic composition. Further improvements in basic materials performance can, however, be obtained by structuring functionality into the ceramics, making functionally gradient materials (FGM). Recently, it has been shown $[18,19]$ that thin layers of controlled porosity can be introduced into the middle of a PMNZTU ceramic wafer by using a tape casting process in which particles of starch are introduced to produce controlled porosity once the tape is sintered. The structure is shown schematically in Fig. 5. Two types of samples were created, with different levels of starch in the central layer. These were labelled 1:1:1 or 1:2:1 according to whether 1 or 2 starch-containing green layers were laminated into the centre of the specimen. Fig. 6 shows a set of cross sections through a sintered 1:2:1 tri-layer. The porous layer is clearly visible. Average porosities for the sintered stacks were measured, as were the porosities of the dense sintered layers (4.2\%) and from these the porosities of the central layers could be calculated. The porous layer has three major effects: firstly it reduces the average relative permittivity of the ceramic rather more quickly than the pyroelectric coefficient; secondly it reduces the volume specific heat in direct proportion to the average porosity; finally, it introduces a layer of lower thermal diffusivity, which acts as a thermal barrier in the system, retaining the heat energy in the pyroelectric element. It has been demonstrated [19] that increases in $\mathrm{F}_{\mathrm{V}}$ of $20 \%$ are achievable, and these can be directly translated into similar improvements in device performance. Fig. 7 shows the measured variation of $F_{V}$ with average porosity in a set of FGM samples. Here, the dashed line is 
a theoretical prediction based on the anticipated effect of the porosity on the dielectric, pyroelectric and thermal properties, which gives:

$$
F_{V}^{A}=p_{D}\left\{1-\frac{3}{2}\left[f_{L} P_{C}+P_{L}\left(1-f_{L}\right)\right]\right\} /\left\{c_{P} \rho_{D} \varepsilon_{D} \varepsilon_{o}\left(1-\frac{3}{2} P_{C}\right)\left(1-\frac{3}{2} P_{L}\right)\right\}
$$

where: $p_{D}=$ pyroelectric coefficient of the dense ceramic

$$
\begin{aligned}
& f_{L}=\text { ratio of porous layer to total ceramic thickness }=t_{L} / T=t_{L} /\left(\left(2 t_{C}+t_{L}\right)\right. \\
& P_{C}=\text { porosity of the outer ceramic layers } \\
& P_{L}=\text { porosity of the porous ceramic layer } \\
& \rho_{D}=\text { theoretical density of ceramic } \\
& c_{p}=\text { specific heat of dense ceramic } \\
& \varepsilon_{D}=\text { relative permittivity of fully-dense ceramic }
\end{aligned}
$$

The fit between theory and experiment is excellent.

\section{Pyroelectric Thin and Thick Films}

The ability to integrate the pyroelectric material as a thin film directly onto the silicon wafer bearing the signal processing readout chips could give major benefits in cost (because no costly ceramic processing and bonding steps would be required) and performance (because the thin films would be more thermally sensitive). However, there are many issues to address. Firstly, the film must be an appropriate thickness for the application. For pyroelectric applications, the ideal thickness is between 0.1 and 1 $\mu \mathrm{m}$ in thickness, depending upon the details of the physical design of the array (e.g. IR 
absorption mechanism, thermal isolation). Secondly, the temperature of film growth must be sufficiently low for the silicon metallization to survive. This is typically $<575^{\circ} \mathrm{C}$ for an array with active silicon components, although it is strongly dependent upon atmosphere [20], and can be considerably lower in air. It can be higher if an inert atmosphere, or a wafer without active circuitry, is used. Thirdly, while the structure (crystallite orientation, porosity) of a film is not necessarily an issue in its own right, it is usually intimately related to the final electrical properties, which are critically important. Finally, film "processability" is very important in determining the ability to fabricate a device. This includes the conformality (or otherwise) of the coating method, the chemical compatibility of the layer with the underlying substrate and electrodes, the techniques available for patterning the film and its general industrial acceptability.

A wide range of Chemical Solution Deposition (CSD) processes have been developed for ferroelectric thin film fabrication. Sol gel methods, in which the metal cations are taken up into solution as alkoxides (for $\mathrm{Zr}$ and $\mathrm{Ti}$ ) or acetates (for $\mathrm{Pb}$ and many dopant ions such as $\mathrm{Mn}$ ) are usually based on chelating solvents such as 2-methoxy ethanol (2ME) [21, 22], acetic acid with alcohols and stabilisers such as acetyl acetone and ethylene glycol (EG) $[23,24]$ and diols [25]. There has been a move to eliminate the use of $2 \mathrm{ME}$, because of its carcinogenic and teratogenic properties, leading to a greater emphasis on the use of simple alcohol solvents such as ethanol or methanol [26].

The fabrication of Mn-doped pyroelectric PZT films is a good example of how CSD can be used for the growth of ferroelectric thin films for MEMS applications [27]. In the particular process used, $\mathrm{Pb}(\mathrm{OAc})_{2} .3 \mathrm{H}_{2} \mathrm{O}$ and $\mathrm{Mn}(\mathrm{OAc})_{2}(1 \mathrm{~mol} \%)$ were dissolved in a methanol /methanolamine $\left(\mathrm{CH}_{3} \mathrm{OH} / \mathrm{MEA}\right)$ mixture. $\operatorname{Zr}\left(\mathrm{O}^{\mathrm{n}} \mathrm{Pr}\right)_{4} \cdot{ }^{\mathrm{n}} \mathrm{PrOH}$ and $\operatorname{Ti}\left(\mathrm{O}^{\mathrm{n}} \mathrm{Bu}\right)_{4}$ were mixed separately and $\mathrm{CH}_{3} \mathrm{CH}_{2} \mathrm{OH}$ and a stoichometric amount of acetic acid were 
added. The $\mathrm{Pb}$ solution was added to the $\mathrm{Zr} / \mathrm{Ti}$ solution and acetic acid was added to adjust the $\mathrm{pH}$ and the PZT concentration to $0.4 \mathrm{M}$. This solution was spun onto $\mathrm{Pt}(111) / \mathrm{Ti} / \mathrm{SiO}_{2} / \mathrm{Si}(100)$ substrates (thickness $\mathrm{Pt} / \mathrm{Ti} / \mathrm{SiO}_{2}=100 / 5 / 500 \mathrm{~nm}$ ) at $3000 \mathrm{rpm}$ for 30s. Each layer was pre-fired on a hot plate set at $200{ }^{\circ} \mathrm{C}$ for 30 s and then further annealed on another hot plate set at $530^{\circ} \mathrm{C}$ for $3 \sim 5 \mathrm{~min}$ in air. The thickness of a single layer was about 70nm, so to obtain thicker films more layers were put down by repeating the above procedure. $\mathrm{Au} / \mathrm{Cr}$ top electrodes were used. Access to the $\mathrm{Pt}$ bottom electrode was obtained by wet-etching a corner of PMZT film. The PMZT thin film was poled by applying an electric field at $90^{\circ} \mathrm{C}$.

Zhang, Whatmore et al $[27,31]$ have reported on the pyroelectric and dielectric properties of PMZT30/70 films annealed at various temperatures up to $560^{\circ} \mathrm{C}$. The dielectric constant and loss tangent of a $1 \%$ Mn-doped film annealed at $530^{\circ} \mathrm{C}$ were 260 and 0.006 at $100 \mathrm{~Hz}$ and 257 and 0.0067 at $1 \mathrm{kHz}$, respectively, while the pyroelectric coefficient was $3 \times 10^{-4} \mathrm{CK}^{-1} \mathrm{~m}^{-2}$. Under similar processing conditions, non-doped PZT30/70 thin films exhibit a dielectric constant of 360 and loss tangent of 0.01 [27]. In comparison with PZT thin film with similar thickness, the Mn doped PZT thin film has a lower dielectric constant and a lower or equivalent loss tangent, which is of great significance in enhancing the performance of an infrared detector. Usually, a PZT30/70 thin film has a pyroelectric coefficient of $1 \sim 2 \times 10^{-4} \mathrm{CK}^{-1} \mathrm{~m}^{-2}$. Doping PZT with 1 mol\% Mn increases the pyroelectric coefficient of the films significantly, leading to almost a factor-of-three improvement in the figure-of-merit $\mathrm{F}_{\mathrm{D}}$. It seems likely that both of these improvements can be ascribed to the fact that the Mn acts as a 'hardening' dopant in the PZT lattice, creating oxygen vacancies and pinning the residual domains. It is likely that the internal bias present in the film due to the addition of Mn acts to 
stabilize the internal polarization, accounting for the increase in the pyroelectric coefficient. The pyroelectric coefficient can be further improved by increasing the film annealing temperature from $530{ }^{\circ} \mathrm{C}$ to $560^{\circ} \mathrm{C}$. At $560^{\circ} \mathrm{C}$ annealing temperature, the dielectric constant and loss of films is virtually the same as those at $530^{\circ} \mathrm{C}$ but the pyroelectric coefficient increased to $3.52 \times 10^{-4} \mathrm{CK}^{-1} \mathrm{~m}^{-2}$, improving the $\mathrm{F}_{\mathrm{D}}$ to $3.85 \mathrm{x} 10^{-}$ ${ }^{5} \mathrm{~Pa}^{-0.5}$, one of the best reported for a PZT-based pyroelectric thin film [31].

The introduction of porosity into films such as these has also been shown to result in a reduction in average permittivity and an increase in the pyroelectric figure-of-merit $\mathrm{F}_{\mathrm{V}}$, by up to $25 \%$ relative to a fully-dense layer [28].

PMNZT type films were fabricated using a low temperature composite sol gel technique [29]. A ceramic producing sol and a ceramic powder are mixed together to produce a slurry which is then spun onto a substrate. Subsequent firing at much reduced temperatures $\left(550-800^{\circ} \mathrm{C}\right)$ converts the sol to an oxide ceramic to form a thick film. Intermediate sol infiltration and pyrolysis can be used to vary the density of the films. To demonstrate the use of the composite sol gel technique for producing thick pyroelectric films it was decided to utilise a simplified sol composition $\left(\mathrm{Pb}_{1.05}\left(\mathrm{Zr}_{82.5} \mathrm{Ti}_{17.5}\right) \mathrm{O}_{3}\right)$ due to the difficulties associated with obtaining a perovskite PMNZTU material using the sol gel process. The composition of the PMNZTU powder was maintained at $\mathrm{Pb}_{1.01}\left(\mathrm{Zr}_{82.5} \mathrm{Ti}_{17.5}\right)_{96.87}\left(\mathrm{Mg}_{1 / 3} \mathrm{Nb}_{2 / 3}\right)_{2.5} \mathrm{U}_{0.63} \mathrm{O}_{3}$. It was found that the pyroelectric properties of such films depended strongly on the amount of infiltrating sol, as shown in Table 2 [30]. However, comparison with Table 1 shows that useful pyroelectric figures-of-merit within a factor of two the bulk materials were obtained. 


\section{Conclusions}

The requirements for pyroelectric materials for use in infra-red detector arrays have been reviewed. The properties of “conventional” pyroelectric ceramics have been shown to not be strongly dependent on the ceramic composition. It has been demonstrated that good control of important properties such as electrical resistivity can be obtained by using off-valent doping. It has been shown how the building-in of functionality through the thickness of a ceramic wafer can give substantial improvements in device performance. Ferroelectric thin films made using the sol gel process offer a route to making fully-integrated pyroelectric materials on silicon, and that, again, the inclusion of controlled porosity into the film can improve the pyroelectric figures-of-merit. Thick films made using a composite sol-gel process can offer pyroelectric performances which, while lower than bulk materials, are still useful.

\section{Acknowledgements}

The financial support of EPSRC and IRISYS Ltd. is gratefully acknowledged. Figure 4 is reprinted with kind permission from Elsevier. Figures 5, 6 and 7 are reprinted with kind permission from Blackwell Publishing. Data in Table 2 is reprinted with kind permission from Springer Science and Business Media. 


\section{References}

[1] R.W. Whatmore: Rep. Prog. Phys., 49 (1986), 1335

[2] R. Watton: Ferroelectrics, 91 (1989), 87

[3] R. Watton, P.N. Dennis, J.P. Gillham, P.A. Manning, M.C. Perkins and M.A. Todd: Proc. SPIE, 2020 (1993), 379

[4] R. Blackwell, S. Geldart, M. Kohin, A. Leary and R. Murphy, Proc. SPIE, 5406 (2004), 422

[5] WWW.IRISYS.co.uk

[6] A. Sixsmith, N Johnson and R.W. Whatmore: J. de Physique, 128 (2005), 153

[7] N. Stogdale, S. Hollock, N. Johnson and N. Sumpter Proc SPIE (2003) Sensors, and Command, Control, Communications and Intelligence (C31) Technologies for Homeland Defense and Law Enforcement II 5071: 465

[8] Mansi MV, Porter SG, Galloway JL and Sumpter N Proc SPIE Infrared Technology and Applications XXVII (2001) 4369: 332

[9] http://www.itbepos.com/pr/12920 
[10]R.W. Whatmore and R. Watton: "Pyroelectric Materials and Devices" in Infrared Detectors and Emitters: Materials and Devices (Kluwer Academic Publishers, The Netherlands) pp 99-148 (2001)

[11] R.W. Whatmore and F.W. Ainger: Proc. SPIE, 395 (1983), 261

[12] R.W. Whatmore and A.J. Bell: Ferroelectrics, 35 (1983), 155

[13] C.P.Shaw, S.Gupta, S.B.Stringfellow, A.Navarro, J.R.Alcock and R.W.Whatmore: J. European Ceram. Soc., 22 (2002), 2123

[14] M. Nakamoto, N. Ichinose, N. Iwase and Y. Yamashita: J. Ceram. Soc. Jpn., 110 (2002), 639

[15] R.W. Whatmore: Ferroelectrics, 49 (1983), 201

[16] S.B. Stringfellow, S. Gupta, C. Shaw, J.R. Alcock and R.W. Whatmore: J. European Ceram. Soc., 22 (2002), 573

[17] R.W. Whatmore, O. Molter and C.P. Shaw: J. European Ceram. Soc., 23 (2003), 721

[18] A. Navarro, R.W. Whatmore and J.R. Alcock: J. Electroceramics, 13 (2004), 413

[19] C.P Shaw, R.W. Whatmore and J.R. Alcock: J. Amer. Ceram. Soc., 90 (2007), 137

[20] P.P. Donohue, M.A. Todd, C.J. Anthony, A.G. Brown, M.A.C. Harper and R. Watton: Integrated Ferroelectrics, 41 (2001), 25 
[21] S.R. Gurkovitch and J.B Blum: Ferroelectrics, 62 (1985), 189

[22] K.D. Budd, S.K. Dey and D.A. Payne: Brit. Ceram. Proc, 36 (1985), 107

[23] G. Yi, Z. Wu and M. Sayer: J. Appl. Phys. 64 (1988), 2717

[24] R.W. Schwartz, T.J. Boyle, S.J. Lockwood, M.B. Sinclair, D. Dimos and C.D. Buchheit: Integrated Ferroelectrics, 7 (1995), 259

[25] N.J. Phillips and S.J. Milne: J. Mater. Chem., 1 (1991), 893

[26] Q. Zhang, R.W. Whatmore and M.E. Vickers: J. Sol. Gel Science \& Technology, 15 (1999), 13

[27] Q. Zhang and R.W. Whatmore: J. Phys. D: Appl. Phys., 34 (2001), 2296

[28] Q. Zhang , C. P. Shaw, Z. Huang and R. W. Whatmore: Integrated Ferroelectrics, 64 (2004), 207

[29] R.A. Dorey, S.B. Stringfellow, R.W. Whatmore: J.Euro.Ceram.Soc., 22 (2002), 2921

[30] R.A. Dorey and R.W. Whatmore: J. Electroceramics, 12 (2004), 191

[31] R.W.Whatmore, Q. Zhang, Z. Huang and R.A. Dorey: Materials Science in Semiconductor Processing, 5 (2002), 65 